\title{
Periodic satellite orbits of the Broucke- Hadjidemetriou-Hénon family of three-body system with unequal masses
}

\section{Xiaoming Li}

Jinan University

Shijun Liao ( $\nabla$ sjliao@sjtu.edu.cn )

Shanghai Jiao Tong University https://orcid.org/0000-0002-2372-9502

\section{Letter}

Keywords: Broucke-Hadjidemetriou-Hénon, $\mathrm{BHH}$, three-body system, $\mathrm{BHH}$ satellites, triple system

Posted Date: August 26th, 2020

DOI: https://doi.org/10.21203/rs.3.rs-55711/v1

License: (c) (i) This work is licensed under a Creative Commons Attribution 4.0 International License.

Read Full License 


\section{Periodic satellite orbits of the Broucke-Hadjidemetriou- Hénon family of three-body system with unequal masses}

Xiaoming $\mathrm{Li}^{1,2}$ and Shijun $\mathrm{Liao}^{3,4}$

${ }^{1}$ Department of Earth, Atmospheric and Planetary Sciences, Massachusetts Institute of Technology, Cambridge, Massachusetts 02139, USA

${ }^{2}$ MOE Key Laboratory of Disaster Forecast and Control in Engineering, School of Mechanics and Construction Engineering, Jinan University, Guangzhou 510632, China

${ }^{3}$ School of Physics and Astronomy, Shanghai Jiaotong University, Shanghai 200240, China

${ }^{4}$ Center of Advanced Computing, School of Naval Architecture, Ocean and Civil Engineering, Shanghai Jiaotong University, Shanghai 200240, China

Triple systems are common and key objects in astronomy. The three-body problem has received much more attention in recent years ${ }^{1-3}$. All observed periodic triple stars systems ${ }^{4-6}$ belong to the Broucke-Hadjidemetriou-Hénon's $(\mathrm{BHH})$ family $^{7-9}$. The BHH orbits are a family of periodic orbits of the three-body system with the simplest topological free group word ${ }^{10}$ $a$, while Janković and Dmitrašinović ${ }^{1}$ gained 58 equal-mass BHH satellite orbits which have free group words $a^{k}(k>1)$, where $k$ is the topological exponent. However, the BHH satellite orbits with equal mass is lack of realistic meaning because they do not exist in practice. Here we present 419743 new BHH orbits and 179253 new BHH satellites $(k>1)$ for three-body system with unequal mass. Especially, 48761 among the 179253 new BHH satellites are stable and have unequal masses. It suggests that these 48761 stable BHH satellites could be found 
by the observation. Besides, for the three-body system with equal mass at a fixed energy, it was demonstrated that the relationship between the angular momentum $(L)$ and topological scaled period $(T / k)$ of the $\mathbf{B H H}$ satellites is the same as that of the BHH orbits ${ }^{1}$. However, we found that this does not hold for the three-body system with unequal mass. Our findings have broad impact for the astrophysical scenario: they could inspire the theoretical and observational study of the triple system, the formation of triple $\operatorname{stars}^{11}$, the gravitational waves pattern $^{12}$ and the gravitational waves observation ${ }^{13}$ of the triple system.

The triple systems are common and key objects in astronomy ${ }^{3}$. It can help us to understand the formation and evolution of multiple star systems ${ }^{11}$. The three-body problem can be traced back to Newton in 1680 s, but is still an open question in astrophysics today, mainly because it is not an integrable system ${ }^{14}$ and besides has the sensitivity dependance on initial condition (SDIC) ${ }^{15}$, i.e. butterfly-effect that broke a new field of scientific research, i.e. chaos. Even today the three-body problem is still one of central issues for scientists ${ }^{3}$. Especially, periodic orbits of triple system play an important role since they are "the only opening through which we can try to penetrate in a place which, up to now, was supposed to be inaccessible", as pointed out by Poincaré ${ }^{15}$. However, since the famous three-body problem was first put forward, only three families of periodic orbits were found in about three hundred years: (1) the Lagrange-Euler family discovered by Lagrange and Euler in the 18th century; (2) the Broucke-Hadjidemetriou-Hénon (BHH) family ${ }^{7-9}$; (3) the figure-eight family, discovered numerically by Moore ${ }^{16}$ in 1993 and then proofed by Chenciner \& Montgomery ${ }^{17}$ in 2000, until 2013 when Šuvakov and Dmitrašinović ${ }^{18}$ numerically found 13 distinct periodic orbits of the three-body system with equal mass. In recent years, numerically search- 
ing for periodic orbits of the three-body system has been received much attention ${ }^{19-23}$. Šuvakov ${ }^{19}$ reported the satellites of the figure-eight periodic orbit with equal mass. Especially, more than six hundred new families of periodic orbits of equal-mass three-body system were found by Li and $\mathrm{Liao}^{20}$ using a new numerical strategy, namely the clean numerical simulation $(\mathrm{CNS})^{24-26}$ that can give the convergent/reliable numerical solution of chaotic systems in a long enough duration. Li et al. ${ }^{21}$ further used the CNS to obtain 1223 new families of periodic orbits of three-body system with two equal-mass bodies. All of these greatly enrich our knowledge of the famous three-body problem.

With the topological classification method ${ }^{10}$, the so-called $\mathrm{BHH}$ orbits have the simplest topology (free group word $w=a$ ), while the BHH satellites have more free group words $w=a^{k}$, where $k$ is the topological exponent. In theory, Janković and Dmitrašinović ${ }^{1}$ numerically gained $58 \mathrm{BHH}$ satellites $(k>1)$ with equal mass, and found that the relationship between the scaleinvariant angular momentum $(L)$ and the topologically rescaled period $(T / k)$ is the same for both of the $\mathrm{BHH}$ orbits $(k=1)$ and satellites $(k>1)$. On the other side, all practically observed periodic triple star systems belong to the $\mathrm{BHH}$ orbits $(k=1)$. This fact raises a question of whether we can observe any $\mathrm{BHH}$ satellites $(k>1)$ in our universe. Unfortunately, all previously reported BHH satellites have equal mass, which are impossible to be observed in practice. Janković and Dmitrašinović ${ }^{1}$ also mentioned the importance of the realistic case of three different masses. In this letter, we will investigate the BHH orbits and satellite orbits with unequal mass.

Let us consider a three-body system in the Newtonian gravitational field. Without loss of 


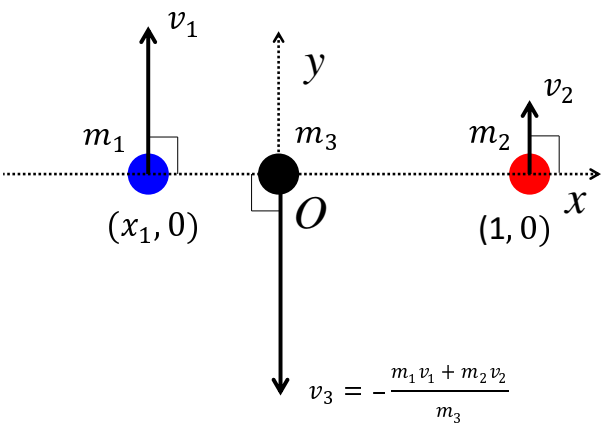

Figure 1: The initial configuration of the three-body system. Here $m_{1}, m_{2}$ and $m_{3}$ denote the mass of body-1, body-2 and body-3, respectively. The corresponding initial velocities are $v_{1}, v_{2}$ and $v_{3}=-\left(m_{1} v_{1}+m_{2} v_{2}\right) / m_{3}$, and the corresponding initial positions are $\left(x_{1}, 0\right),(1,0)$ and $(0,0)$.

generality, let the Newtonian gravitational constant $G=1$. As shown in Figure 1, the three bodies have collinear initial configuration for the BHH family of periodic orbits: $\boldsymbol{r}_{1}(0)=\left(x_{1}, 0\right)$, $\boldsymbol{r}_{2}(0)=\left(x_{2}, 0\right), \boldsymbol{r}_{3}(0)=\left(x_{3}, 0\right)$, and their initial velocities are orthogonal to the line determined by the three bodies: $\dot{\boldsymbol{r}}_{1}(0)=\left(0, v_{1}\right), \dot{\boldsymbol{r}}_{2}(0)=\left(0, v_{2}\right), \dot{\boldsymbol{r}}_{3}(0)=\left(0, v_{3}\right)$.

Due to the homogeneity of the potential field of the three-body system, there is a scaling law : $\boldsymbol{r}^{\prime}=\alpha \boldsymbol{r}, \boldsymbol{v}^{\prime}=\boldsymbol{v} / \sqrt{\alpha}, t^{\prime}=\alpha^{3 / 2} t$, energy $E^{\prime}=E / \alpha$ and angular momentum $L^{\prime}=\sqrt{\alpha} L$. The known periodic orbits of the BHH family and their satellites with equal mass ${ }^{1,7,9}$ have zero total momentum, i.e., $m_{1} \dot{\boldsymbol{r}_{\mathbf{1}}}+m_{2} \dot{\boldsymbol{r}_{\mathbf{2}}}+m_{3} \dot{\boldsymbol{r}_{\mathbf{3}}}=0$. Using the scaling law, we can transform the initial conditions of the known periodic orbits of the BHH family and their satellites to the initial positions

$$
\boldsymbol{r}_{1}(0)=\left(x_{1}, 0\right), \quad \boldsymbol{r}_{2}(0)=(1,0), \quad \boldsymbol{r}_{3}(0)=(0,0)
$$


(a)

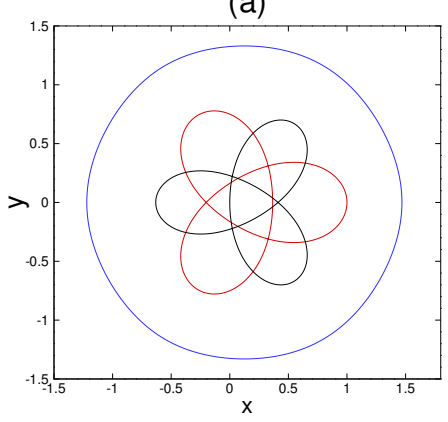

(d)

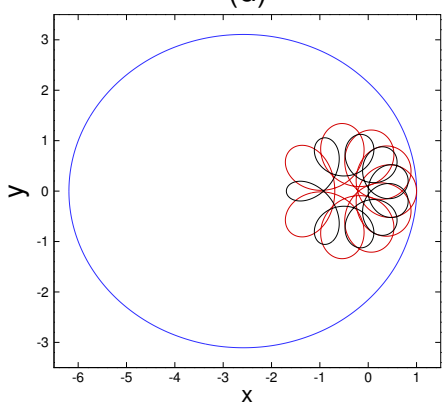

(b)

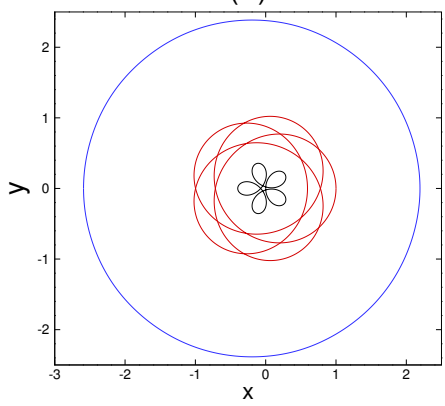

(e)

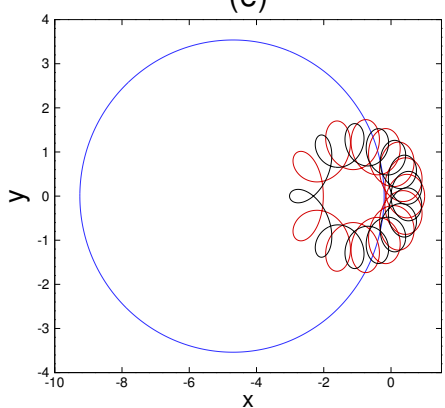

(c)

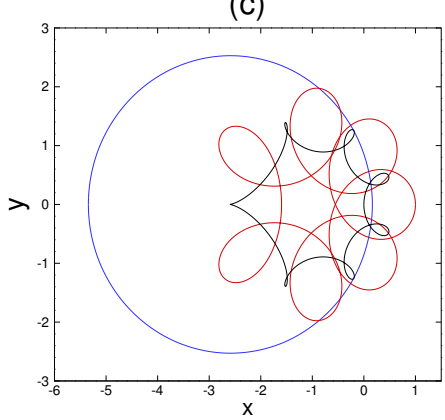

(f)

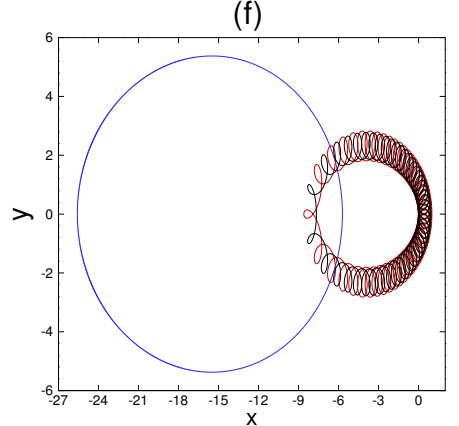

Figure 2: The stable $\mathrm{BHH}$ satellites $(k>1)$ of the three-body system with unequal mass in a rotating system. Blue line: body-1; red line: body-2; black line: body-3. The corresponding physical parameters are given in Table 1.

and the initial velocities

$$
\dot{\boldsymbol{r}}_{1}(0)=\left(0, v_{1}\right), \quad \dot{\boldsymbol{r}}_{2}(0)=\left(0, v_{2}\right), \quad \dot{\boldsymbol{r}}_{3}(0)=\left(0,-\frac{m_{1} v_{1}+m_{2} v_{2}}{m_{3}}\right) .
$$

We use the numerical continuation method ${ }^{27}$ and clean numerical simulation ${ }^{24-26}$ (see Methods) to gain the BHH orbits $(k=1)$ and their satellites $(k>1)$ with unequal mass. Note that all of the known BHH orbits $(k=1)$ and satellites $(k>1)$ are "relative periodic orbits": after a period, these relative periodic orbits will return to initial conditions in a rotating frame of reference. So, there is an individual rotation angle $\theta$ for each relative periodic orbit. 
Table 1: Initial conditions and periods $T$ of some $\mathrm{BHH}$ satellites of three-body system with unequal mass in case of $\boldsymbol{r}_{1}(0)=\left(x_{1}, 0\right), \boldsymbol{r}_{2}(0)=(1,0), \boldsymbol{r}_{3}(0)=(0,0), \dot{\boldsymbol{r}}_{1}(0)=\left(0, v_{1}\right)$, $\dot{\boldsymbol{r}}_{2}(0)=\left(0, v_{2}\right), \dot{\boldsymbol{r}}_{3}(0)=\left(0,-\left(m_{1} v_{1}+m_{2} v_{2}\right) / m_{3}\right)$. Here $m_{i}, x_{i}$ and $v_{i}$ are the mass, initial position and velocity of the $i$ th body, $\theta$ is the rotation angle of relative periodic orbits, and $k$ is the topological power of periodic orbits, respectively.

\begin{tabular}{cccccccccc}
\hline No. & $m_{1}$ & $m_{2}$ & $m_{3}$ & $x_{1}$ & $v_{1}$ & $v_{2}$ & $T$ & $\theta$ & $k$ \\
\hline (a) & 0.44 & 0.87 & 1 & -1.219929 & -0.992252 & -0.513024 & 9.175828 & 0.500326 & 3 \\
(b) & 0.1 & 0.2 & 1 & -2.590389 & -0.619538 & -0.865730 & 23.182211 & 0.110300 & 5 \\
(c) & 0.64 & 0.36 & 1 & -5.343038 & -0.320962 & -0.737472 & 36.396579 & 0.085297 & 7 \\
(d) & 0.4 & 0.7 & 1 & -6.190951 & -0.330208 & -0.703472 & 40.846418 & 0.107928 & 9 \\
(e) & 0.6 & 0.8 & 1 & -9.253167 & -0.235487 & -0.683551 & 60.335058 & 0.086984 & 13 \\
(f) & 0.82 & 0.9 & 1 & -25.585414 & -0.093121 & -0.673916 & 204.731304 & 0.056233 & 48 \\
\hline
\end{tabular}


(a)

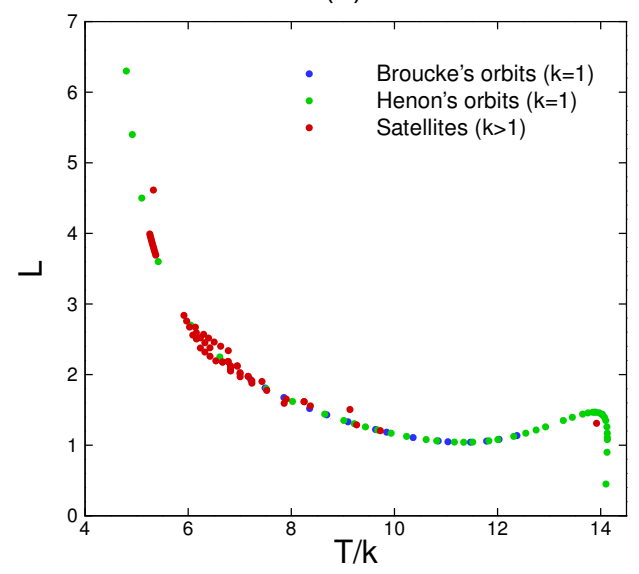

(c)

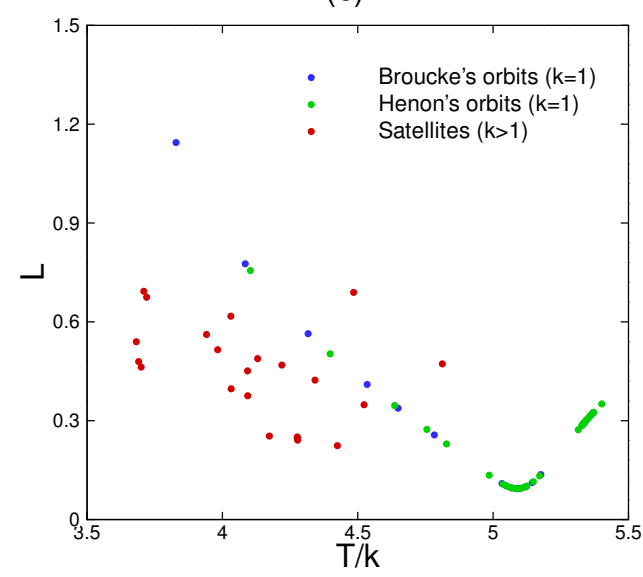

(b)

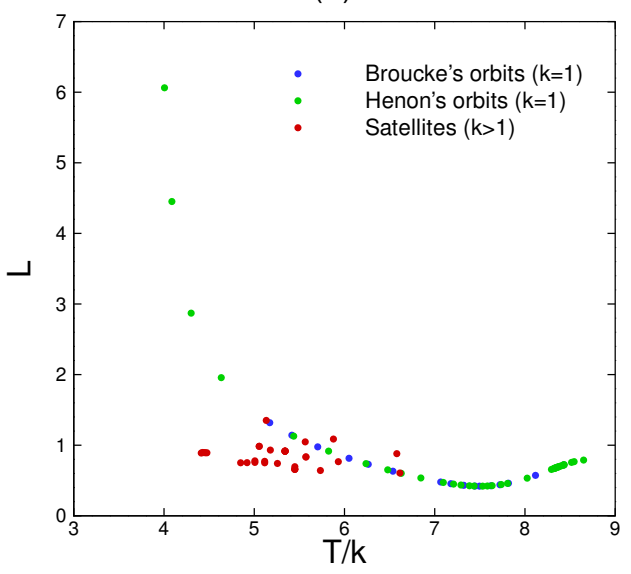

(d)

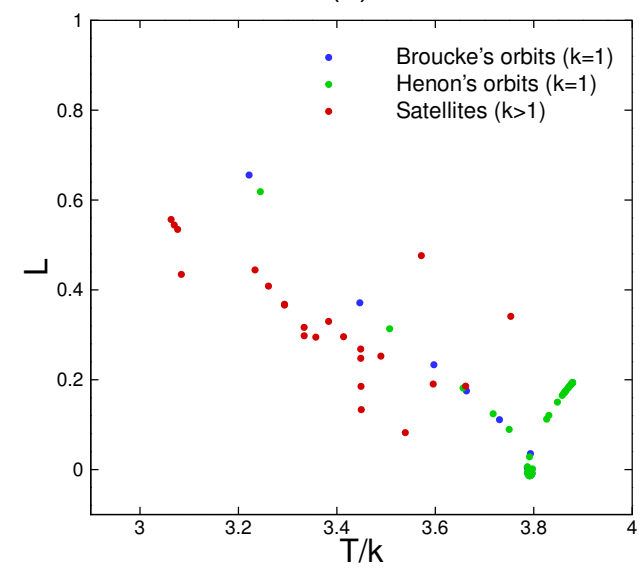

Figure 3: The angular momentum $(L)$ versus the topological rescaled period $(T / k)$ for $\mathrm{BHH}$ periodic orbits and their satellites at fixed energy $E=-1 / 2$ with different mass: (a) $m_{1}=m_{2}=$ $m_{3}=1$; (b) $m_{1}=0.7, m_{2}=0.9, m_{3}=1$; (c) $m_{1}=0.5, m_{2}=0.8, m_{3}=1$; (d) $m_{1}=0.4$, $m_{2}=0.7, m_{3}=1$. 
Starting from the 16 known Broucke's periodic orbits $(k=1 \text { with equal mass })^{7}$, the 45 known Hénon's periodic orbits $(k=1 \text { with equal mass })^{9}$ and the 58 known BHH satellites $(k>1$ with equal mass) ${ }^{1}$, we respectively obtain 124780, 294963 and 179253 new periodic orbits of the three-body system with unequal mass for $m_{1} \in[0.1,1), m_{2} \in[0.1,1)$ and $m_{3}=1$. Totally, we gain 419743 new BHH (relative periodic) orbits ( $k=1$ with unequal mass) and 179253 new BHH (relative periodic) satellites ( $k>1$ with unequal mass). Note that all of them are retrograde, say, the binary system and the third body move in opposite direction. Besides, these orbits are relatively periodic, say, the orbits are closed curves in a rotating frame of reference. The corresponding initial conditions, the periods $T$ and the rotation angles $\theta$ of these new BHH orbits $(k=1)$ and satellites $(k>1)$ with unequal mass are given in the corresponding supplementary files. The return distance of these periodic orbits and satellites satisfies

$$
d=\sqrt{\sum_{i=1}^{3}\left(\left(\boldsymbol{r}_{i}(T)-\boldsymbol{r}_{i}(0)\right)^{2}+\left(\dot{\boldsymbol{r}}_{i}(T)-\dot{\boldsymbol{r}}_{i}(0)\right)^{2}\right)}<10^{-10}
$$

in a rotating frame of reference, where $T$ is the period. Note that Broucke and Boggs ${ }^{28}$ gave dozens of the BHH orbits $(k=1)$ with unequal mass (their ratios of mass are different from ours), but neither have any BHH satellites $(k>1)$ with unequal mass been reported, to the best of our knowledge. Note that, among our newly-found 598996 BHH orbits and satellites with unequal mass, there are 151925 stable BHH orbits $(k=1)$ and 48761 stable BHH satellites $(k>1)$. Here, it should be emphasized that these stable $48761 \mathrm{BHH}$ satellites have unequal masses and thus many among them could be observed in practice. The stability of these periodic orbits and satellites is marked by "S" in the corresponding supplementary files. For examples, six new BHH satellites with unequal mass are shown in Figure 2. All of the six BHH relatively periodic satellites are 
linearly stable. Their initial conditions, periods and topological powers $(k)$ are listed in Table 1. It should be emphasized that we can modify these 200686 stable new BHH orbits and satellites with unequal mass to an arbitrary accuracy by means of the above-mentioned numerical strategy and the CNS. For example, we further obtained much more accurate initial conditions of the six BHH relatively periodic satellites (listed in Table 1) with return distance $d<100^{-100}$, as shown in the corresponding supplementary file. From the viewpoint of accuracy, all of these numerical solutions have no essential difference from "closed-form" analytic solutions that however unfortunately do not exist for three-body problem in general.

With rescaling to the same energy $E=-1 / 2$, Janković and Dmitrašinović ${ }^{1}$ found that, in case of equal mass, the relationship between the scale-invariant angular momentum $(L)$ and the topologically rescaled period $(T / k)$ is the same for both of the $\mathrm{BHH}$ orbits $(k=1)$ and satellites $(k>1)$, as shown in Figure 3(a), where $k$ is the topological exponent of periodic orbits. However, for our newly-found periodic orbits with unequal masses (at the same energy $E=-1 / 2$ ), it is found that the relationship between the scale-invariant angular momentum $(L)$ and period $(T / k)$ of the $\mathrm{BHH}$ satellites $(k>1)$ is different from that of the $\mathrm{BHH}$ orbits $(k=1)$, as illustrated in Figure 3 (b)-(d). It suggests that the relationship between the scale-invariant angular momentum $(L)$ and topologically rescaled period $(T / k)$ of the BHH orbits $(k=1)$ and satellites $(k>1)$ should be more complicated in general cases of unequal masses $m_{1} \neq m_{2} \neq m_{3}$ than in the case of the equal mass $m_{1}=m_{2}=m_{3}$.

In 2016 Janković and Dmitrašinović ${ }^{1}$ numerically gained 58 BHH satellites $(k>1)$ with 
equal mass, and mentioned the importance of the realistic case of three different masses in their open questions. In this letter, we numerically found 419743 new BHH (relative periodic) orbits $(k=1)$ and 179253 new $\mathrm{BHH}$ (relative periodic) satellites $(k>1)$ for three-body system with unequal mass, which have never been reported. Especially, 48761 (about 27.2\%) among the 179253 new BHH (relative periodic) satellites $(k>1)$ are stable and have unequal mass. It should be emphasized that all practically observed periodic triple star systems belong to the family of BHH orbits $(k=1)$, but up to now $\mathrm{BHH}$ (relative periodic) satellites $(k>1)$ with unequal mass were never reported before even in theory. Therefore, these 48761 stable $\mathrm{BHH}$ (relative periodic) satellites $(k>1)$ with unequal masses have important meaning in practice: many among them could be found by the observation. Besides, for the three-body system with equal mass at a fixed energy, Janković and Dmitrašinović ${ }^{1}$ numerically demonstrated that the relationship between the angular momentum $(L)$ and topological period $(T / k)$ of the $\mathrm{BHH}$ satellites $(k>1)$ is the same as that of the $\mathrm{BHH}$ orbits $(k=1)$. However, we found that this does not hold for the three-body system with unequal mass. Therefore, our study has important meanings not only in practice but also in theory: they could greatly deepen our understandings and enrich our knowledge for the practical observation of the $\mathrm{BHH}$ satellites $(k>1)$, formation of multiple stars, the gravitational waves pattern, the gravitational waves observation of the triple system, and so on. 


\section{Methods}

Briefly speaking, the numerical continuation method can be used to gain solution of a differential system

$$
\dot{\boldsymbol{u}}=F(\boldsymbol{u}, \lambda)
$$

where $\lambda$ a physical parameter, called "natural parameter". Assume that $\boldsymbol{u}_{0}$ is a solution at a natural parameter $\lambda=\lambda_{0}$. Using the solution $\boldsymbol{u}_{0}$ at $\lambda=\lambda_{0}$ as an initial guess, a new solution $\boldsymbol{u}^{\prime}$ can be obtained at a new natural parameter $\lambda=\lambda_{0}+\Delta \lambda$ through the Newton-Raphson method ${ }^{29,30}$ and the clean numerical simulation $(\mathrm{CNS})^{24-26}$, if the increment $\Delta \lambda$ is small enough to make sure

iterations convergence. Note that the CNS ${ }^{24-26}$ is a numerical strategy to obtain reliable numerical simulation of chaotic systems in a given time of interval. The CNS is based on an arbitrary high order Taylor series method ${ }^{31,32}$ and the multiple precision arithmetic ${ }^{33}$, plus a convergence check using an additional computation with even smaller numerical error. The CNS ${ }^{24-26}$ is used here mainly because three-body system is chaotic in general.

First of all, using the known BHH orbits $(k=1)$ and satellites $(k>1)$ with equal mass ( $\left.m_{1}=m_{2}=m_{3}=1\right)$ as initial guesses and $m_{1}$ as a natural parameter of the continuation method, we obtain new periodic orbits with various $m_{1}$ by continually correcting the initial conditions $x_{1}$, $v_{1}, v_{2}$, the period $T$ and the rotation angle $\theta$. Then, using these periodic solutions with $m_{1} \neq 1$, $m_{2}=m_{3}=1$ as initial guesses and $m_{2}$ as a natural parameter of the continuation method, we similarly gain periodic orbits for different values of $m_{2}$. In this way, we can obtain the corresponding $\mathrm{BHH}$ (relative periodic) orbits $(k=1)$ and satellites $(k>1)$ with unequal mass $m_{1} \neq m_{2} \neq m_{3}$, 
where $m_{3}=1$.

1. Janković, M. R. \& Dmitrašinović, V. Angular momentum and topological dependence of Kepler's third law in the broucke-hadjidemetriou-hénon family of periodic three-body orbits. Phys. Rev. Lett. 116, 064301 (2016).

2. Archibald, A. M. et al. Universality of free fall from the orbital motion of a pulsar in a stellar triple system. Nature 559, 73-76 (2018).

3. Stone, N. C. \& Leigh, N. W. A statistical solution to the chaotic, non-hierarchical three-body problem. Nature 576, 406-410 (2019).

4. Dimitrov, W. et al. The hierarchical triple system dy lyncis. Monthly Notices of the Royal Astronomical Society 466, 2-10 (2017).

5. Marcadon, F., Appourchaux, T. \& Marques, J. Asteroseismic and orbital analysis of the triple star system HD 188753 observed by Kepler. Astronomy \& Astrophysics 617, A2 (2018).

6. Torres, G., Stefanik, R. P. \& Latham, D. W. Dynamical masses for the triple system hd 28363 in the hyades cluster. The Astrophysical Journal 885, 9 (2019).

7. Broucke, R. On relative periodic solutions of the planar general three-body problem. Celestial Mechanics 12, 439 - 462 (1975).

8. Hadjidemetriou, J. D. The stability of periodic orbits in the three-body problem. Celestial Mechanics 12, 255-276 (1975). 
9. Hénon, M. A family of periodic solutions of the planar three-body problem, and their stability. Celestial mechanics 13, 267-285 (1976).

10. Montgomery, R. The $\mathrm{n}$-body problem, the braid group, and action-minimizing periodic solutions. Nonlinearity 11, 363 (1998).

11. Reipurth, B. \& Mikkola, S. Formation of the widest binary stars from dynamical unfolding of triple systems. Nature 492, 221-224 (2012).

12. Dmitrašinović, V., Šuvakov, M. \& Hudomal, A. Gravitational waves from periodic three-body systems. Phys. Rev. Lett. 113, 101102 (2014).

13. Meiron, Y., Kocsis, B. \& Loeb, A. Detecting triple systems with gravitational wave observations. The Astrophysical Journal 834, 200 (2017).

14. Musielak, Z. E. \& Quarles, B. The three-body problem. Rep. Prog. Phys. 77, 065901 (30pp) (2014).

15. Poincaré, J. H. Sur le probléme des trois corps et les équations de la dynamique. Divergence des séries de m. Lindstedt. Acta Math. 13, 1-270 (1890).

16. Moore, C. Braids in classical dynamics. Phys. Rev. Lett. 70, 3675-3679 (1993).

17. Chenciner, A. \& Montgomery, R. A remarkable periodic solution of the three-body problem in the case of equal masses. Annals of Mathematics 152, 881-901 (2000).

18. Šuvakov, M. \& Dmitrašinović, V. Three classes of newtonian three-body planar periodic orbits. Phys. Rev. Lett. 110, 114301 (2013). 
19. Šuvakov, M. Numerical search for periodic solutions in the vicinity of the figure-eight orbit: slaloming around singularities on the shape sphere. Celestial Mechanics and Dynamical Astronomy 119, 369-377 (2014).

20. Li, X. \& Liao, S. More than six hundred new families of newtonian periodic planar collisionless three-body orbits. SCIENCE CHINA Physics, Mechanics \& Astronomy 60, 129511 (2017).

21. Li, X., Jing, Y. \& Liao, S. Over a thousand new periodic orbits of a planar three-body system with unequal masses. Publications of the Astronomical Society of Japan 70, 64 (2018).

22. Dmitrašinović, V., Hudomal, A., Shibayama, M. \& Sugita, A. Linear stability of periodic three-body orbits with zero angular momentum and topological dependence of Kepler's third law: a numerical test. Journal of Physics A: Mathematical and Theoretical 51, 315101 (2018).

23. Li, X. \& Liao, S. Collisionless periodic orbits in the free-fall three-body problem. New Astronomy 70, 22-26 (2019).

24. Liao, S. On the reliability of computed chaotic solutions of non-linear differential equations. Tellus A 61, 550-564 (2009).

25. Liao, S. \& Wang, P. On the mathematically reliable long-term simulation of chaotic solutions of lorenz equation in the interval [0,10000]. Sci. China - Phys. Mech. Astron. 57, 330 - 335, (2014).

26. Hu, T. \& Liao, S. On the risks of using double precision in numerical simulations of spatiotemporal chaos. Journal of Computational Physics 418, 109629 (2020). 
27. Allgower, E. L. \& Georg, K. Introduction to numerical continuation methods, vol. 45 (SIAM, 2003).

28. Broucke, R. \& Boggs, D. Periodic orbits in the planar general three-body problem. Celestial mechanics 11, 13-38 (1975).

29. Farantos, S. C. Methods for locating periodic orbits in highly unstable systems. Journal of Molecular Structure: THEOCHEM 341, 91 - 100 (1995).

30. Lara, M. \& Pelaez, J. On the numerical continuation of periodic orbits - an intrinsic, 3dimensional, differential, predictor-corrector algorithm. Astronomy and Astrophysics 389, 692-701 (2002).

31. Chang, Y. F. \& Corhss, G. F. ATOMFT: Solving ODEs and DAEs using Taylor series. Computers Math. Applic. 28, 209 - 233 (1994).

32. Barrio, R., Blesa, F. \& Lara, M. VSVO formulation of the Taylor method for the numerical solution of ODEs. Computers \& Mathematics with Applications 50, 93-111 (2005).

33. Fousse, L., Hanrot, G., Lefèvre, V., Pélissier, P. \& Zimmermann, P. Mpfr: A multiple-precision binary floating-point library with correct rounding. ACM Transactions on Mathematical Software (TOMS) 33, 13-es (2007).

Acknowledgements This work was carried out on TH-1A (in Tianjin) and TH-2 (in Guangzhou) at National Supercomputer Center, China. It is partly supported by National Natural Science Foundation of China 
(Approval No. 91752104), China Postdoctoral Science Foundation (Approval No. 2020M673058) and International Program of Guangdong Provincial Outstanding Young Researcher.

Author contributions X.M.L. calculated the periodic orbits and generated the first draft. S.J.L. analysed the results and revised the letter. All authors contributed to the discussion and revision of the final manuscript.

Competing Interests The authors declare that they have no competing financial interests.

Correspondence Correspondence and requests for materials should be addressed to S.J.L. (sjliao@ sjtu.edu.cn). 


\section{Figures}

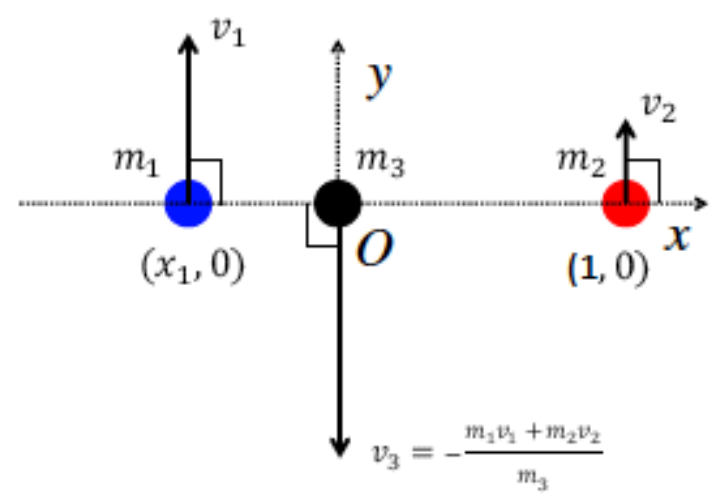

\section{Figure 1}

The initial configuration of the three-body system. Here $\mathrm{m} 1, \mathrm{~m} 2$ and $\mathrm{m} 3$ denote the mass of body-1, body2 and body-3, respectively. The corresponding initial velocities are $v 1, v 2$ and $v 3=-(m 1 v 1+m 2 v 2) / m 3$, and the corresponding initial positions are $(x 1 ; 0),(1,0)$ and $(0,0)$. generality, let the Newtonian gravitational constant $G=1$. As shown in Figure 1 , the three bodies have collinear initial configuration for the BHH family of periodic orbits: $r 1(0)=(x 1 ; 0), r 2(0)=(x 2,0), r 3(0)=(x 3,0)$, and their initial velocities are orthogonal to the line determined by the three bodies: $\nabla 1(0)=(0, v 1), \otimes(0)=(0, v 2), \otimes(0)=(0, v 3)$.

(a)

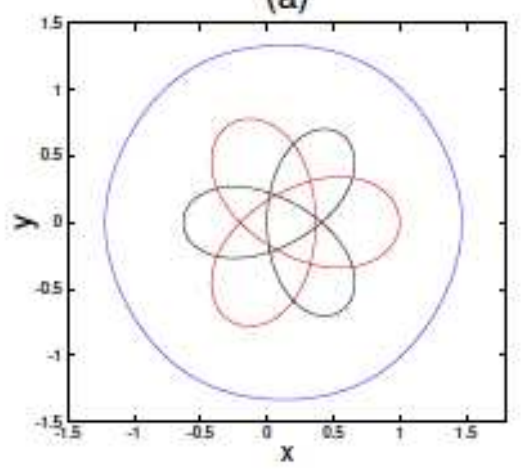

(d)

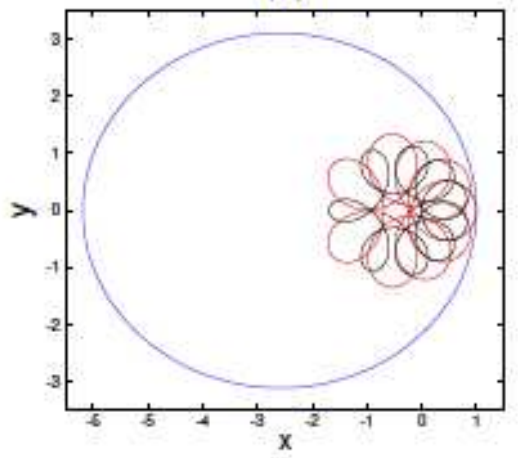

(b)

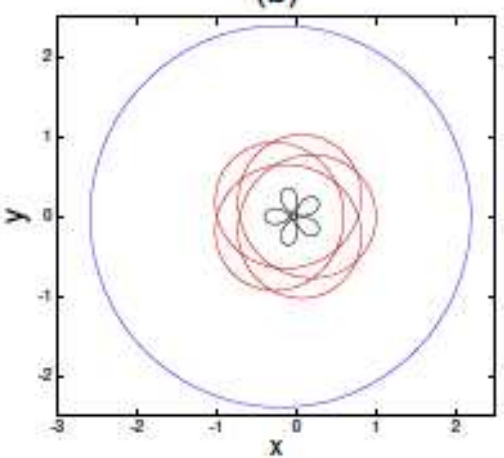

(e)

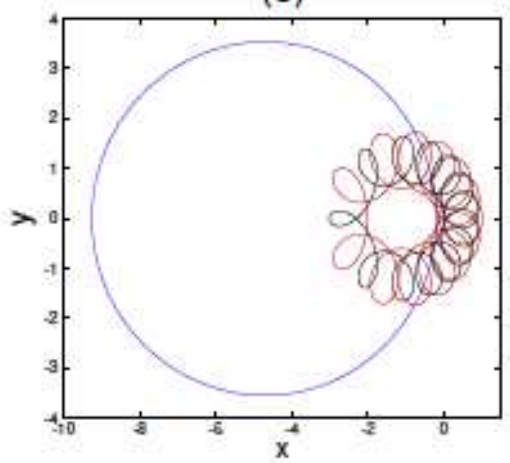

(c)

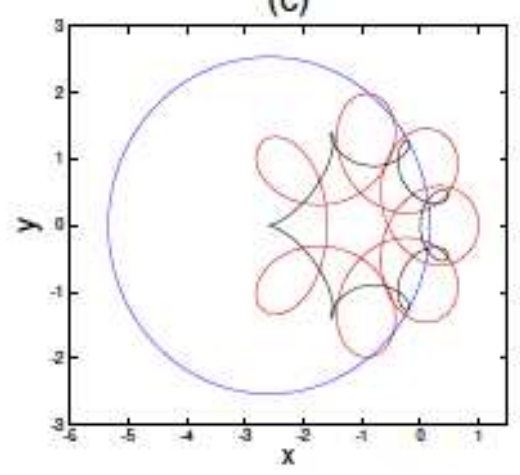

(f)

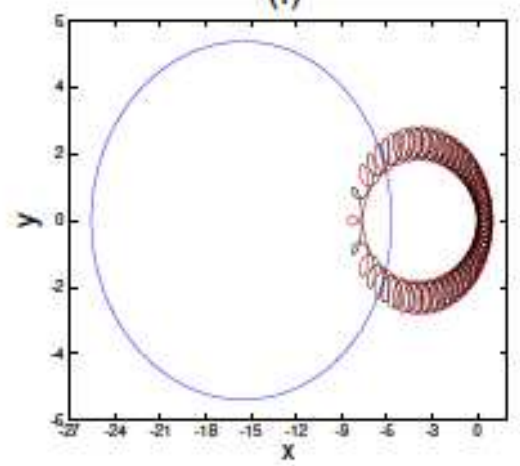


Figure 2

The stable BHH satellites $(k>1)$ of the three-body system with unequal mass in a rotating system. Blue line: body-1; red line: body-2; black line: body-3. The corresponding physical parameters are given in Table 1.

(a)

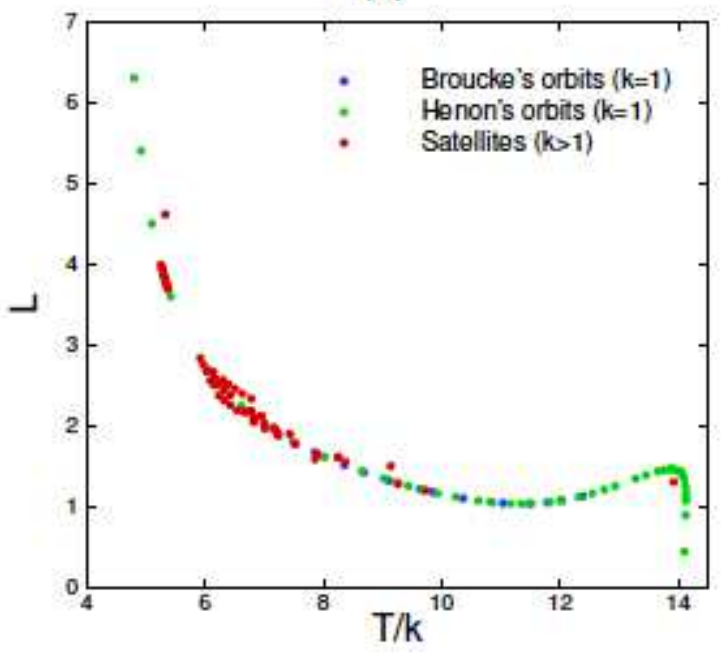

(c)

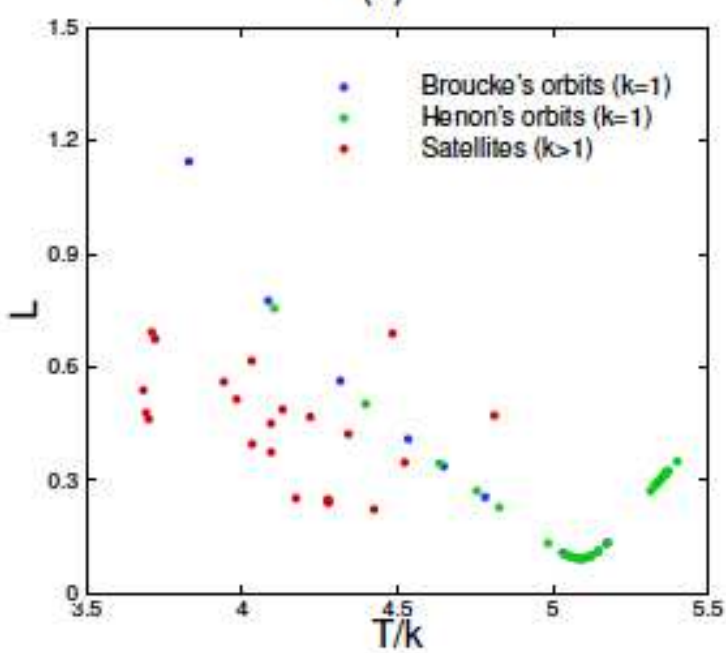

(b)

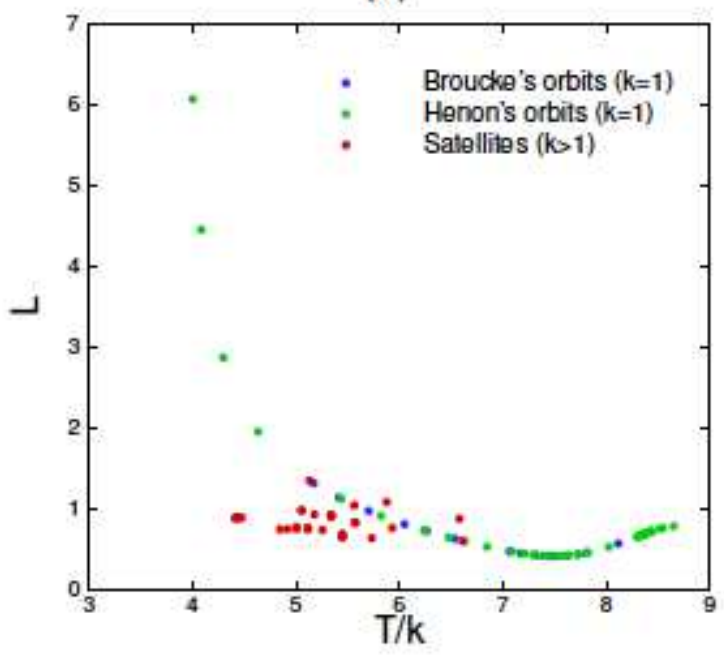

(d)

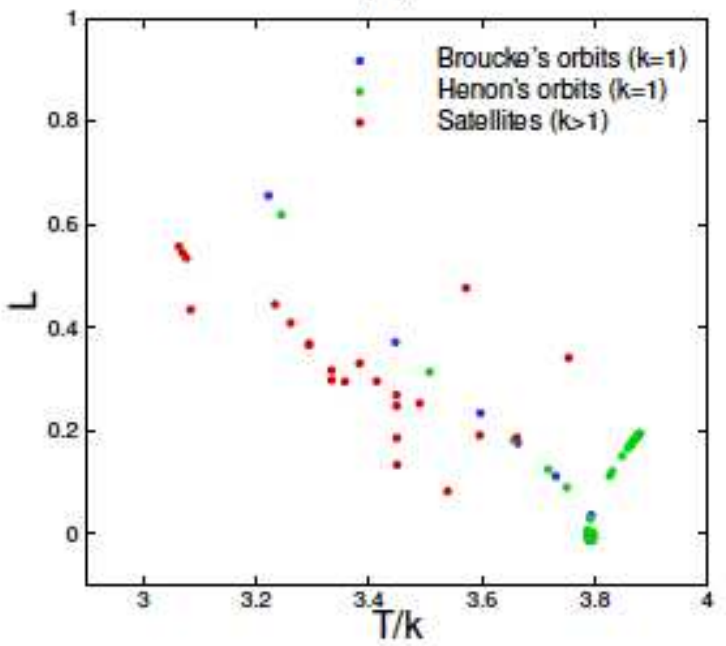

\section{Figure 3}

The angular momentum $(L)$ versus the topological rescaled period $(T=k)$ for $B H H$ periodic orbits and their satellites at fixed energy $E=-1 / 2$ with different mass: (a) $m 1=m 2=m 3=1 ;$ (b) $m 1=0: 7, m 2=0: 9, m 3=$ $1 ;(\mathrm{c}) \mathrm{m} 1=0: 5, \mathrm{~m} 2=0: 8, \mathrm{~m} 3=1 ;$ (d) $\mathrm{m} 1=0.4, \mathrm{~m} 2=0.7, \mathrm{~m} 3=1$.

\section{Supplementary Files}

This is a list of supplementary files associated with this preprint. Click to download. 
- Supplementarysixexamples.txt

- SupplementaryBroucke.txt

- SupplementaryHenon.txt

- SupplementarySatellites.txt 